\title{
Role of Dynamic Contrast Enhanced Magnetic Resonance Imaging and Mammogram in Evaluation of Breast Lesions with Pathological Correlation
}

\author{
K Harsha Veena ${ }^{\circledR}$, Ramakrishna Rao Baru ${ }^{\odot 2}$ \\ ${ }^{1}$ Post Graduate, Department Of Radiodiagnosis, Osmania Medical College Hyderabad, Hyderabad, Telangana, India, ${ }^{2}$ Professor, Department of Radio-Diagnosis, \\ Narayana Medical College \& Hospital, Nellore, Andhra Pradesh, India.
}

\section{Abstract}

Background: To perform mammography and dynamic contrast enhanced MRI in patients with clinically suspicious breast lumps and correlating the findings with pathology in patients of positive imaging. Subjects and Methods: total of 40 patients evaluated through MRI BI-RADS lexicon (General Electric Medical Systems) at 1.5T in combination with dynamic kinetic analysis of time and signal curves for lesion characterization. Results: The mean age was 44 years, maximum of patients $(n=22)$ belongs to age group of 41-60 years $(52.5 \%)$. Five lesions showed dark internal septations, as benign. 11 lesions (33.5\%) showed heterogenous enhancement, as malignant. Early rim enhancement is observed in 3 lesions (9\%). Out of which two of them were proven to be malignant and one lesion was an infective abscess. Invasive Duct Cell Carcinoma was the common histology in the malignant lesions, observed in 13 out of $18(72.2 \%)$. Fibroadenoma was the common benign breast lesion, observed in 8 out of $19(42.1 \%)$. In 4 post operative patients, $2(50 \%)$ were post operative fibrosis, $1(25 \%)$ was recurrence and $1(25 \%)$ was post operative collection. In 18 malignant lesions, 11 patients $(61.1 \%)$ had skin retraction, 10 patients $(55.5 \%)$ had skin thickening, 9 patients (50\%) had axillary lymphadenopathy, 2 patients (11.1\%) had nipple retraction, and one patient (5.6\%) had pectoralis involvement. In our study, MR imaging alone has Sensitivity of $88.8 \%$, specificity of $86.3 \%$, PPV of $84.2 \%$, and NPV of $90.4 \%$ with Accuracy $87.5 \%$. Conclusion : The dynamic contrast enhanced MRI is important tool to evaluate the postoperative breast in differentiating between benign postoperative changes. By combining kinetic analysis with morphological analysis it add benefit to categorise the breast lesions into benign and malignant with increased confidence levels. CE-MRI with both morphology and kinetic curve assessment has a Sensitivity of $94.5 \%$, Specificity of $100 \%$ with Accuracy of $97.5 \%$.

Keywords: Lymphadenopathy, Breast cancer, mammography, Dynamic contrast enhanced MRI, Positive Predictive Value.

Corresponding Author: Ramakrishna Rao Baru, Professor, Department of Radio-Diagnosis, Narayana Medical College \& Hospital, Nellore, Andhra Pradesh, India.

E-mail: drkdurga60@gmail.com

Received: 20 August 2020

Revised: 03 October 2020

Accepted: 11 October 2020

Published: 31 December 2020

\section{Introduction}

Breast cancer is leading cancer mortality in women. Currently screen film x-ray mammography, real time ultrasound, color Doppler and MRI are being utilised for detection of breast cancer. Although mammography is the best diagnostic method for early detection, problems still exist. Even with high quality screening, about $40 \%$ of carcinomas are detected only when they are larger than $1.5 \mathrm{cms}$, and $20 \%$ to $40 \%$ of the carcinomas become apparent in the interval between screenings. ${ }^{[1,2]}$

The limitations of the $\mathrm{x}$ ray mammography have led to development of complimentary imaging techniques. The most accepted adjunct modality is breast sonography which is widely used in diagnostic evaluation of women with abnormal screening mammography or clinical exams. ${ }^{[3]}$ However, in the case of small lesions, sonography is not sensitive and specific enough to rule out malignant disease.

Dynamic contrast material enhanced MRI of breast with gadolinium based contrast is well accepted. Breast carcinomas generally show faster and stronger signal intensity increase after a bolus injection of gadolinium.

Dynamic contrast enhanced MRI is a very sensitive method and have capable of detecting even small lesions. Effective results may achieved when contrast enhanced MRI was used as an additional modality in cases with significant risk of breast cancer and where assessment by conventional imaging was equivocal. Early contrast enhanced MR imaging studies showed marked increased signal intensity in cancers compared 
to surrounding fibro-glandular tissue, with sensitivities $100 \%$ for invasive disease. ${ }^{[4]}$

Initial reports regarding use of dynamic contrast enhanced MR imaging to measure increased uptake of contrast in suspicious breast lesion revealed specificity of $30-85 \%$ and sensitivity of $90-99 \%{ }^{[5]}$ However limited literature exists as regards application of this technique Vis a Vis sonomammography. The current study designed to prove the usefulness of dynamic contrast enhanced MRI as compared to sonomammography in the evaluation of suspicious breast lesions.

\section{Subjects and Methods}

\section{Source of data}

Patients referred to mammography unit in department of Radiodiagnosis of Osmania General Hospital, Hyderabad for screening or clinically suspicious breast lumps. All the patients are evaluated according to MRI BI-RADS lexicon in combination with dynamic kinetic analysis of time/signal intensity curves for lesion characterization as benign or malignant.

Time period: December 2015 to January 2018

Sample size: 40 patients

Type of study: prospective

\section{Inclusion criteria}

1. Patients with clinically suspicious breast lumps.

2. Patients with family history of breast neoplasms.

\section{Exclusion criteria}

1. Patients with contraindications to MRI: patients with ferromagnetic implants, claustrophobia etc

2. Patients with pre-existing spine deformities and physically disabled patients.

3. Male patients.

\section{Data acquisition}

Informed consent will be taken from all the patients.

All the MRI examinations are performed on echo speed system (General Electric Medical Systems) at 1.5T. Imaging of all patients in prone position in a dedicated double breast coil. Compression device is used.

\section{The following sequences were obtained.}

1. A transverse $\mathrm{T} 1 \mathrm{~W}$ spin-echo sequence was performed for localization purposes.

2. Axial T2W fast spin echo sequences

3. Fat suppressed T2W fast spin-echo sequence
4. A 3D axial fat suppressed $\mathrm{T} 1 \mathrm{~W}$ fast gradient is recalled echo sequence was obtained before, then 6 sets of images after bolus injection of $0.1 \mathrm{mmol} / \mathrm{kg}$ of gadodiamide with an acquisition time 60 seconds for each set for 84 images.

Fat suppression and subtraction of pre contrast from the first set of post contrast images was done.

Morphologic analysis done on post processed subtracted images using MRI BI-RADS Lexicon (American College of Radiology) and visual kinetic analysis of time signal intensity was done.

\section{Results}

A random study of 40 patients done in our institute to evaluate the diagnostic value of DCE-MRI in breast lesions. All these patients were clinically suspected to have breast lesions. The mean age is 44 years. The maximum of patients $(n=22)$ were in the age group of $41-60$ years $(52.5 \%)$. There is only one patient below the age of 20 years.

35 patients $(87.5 \%)$ presented as mass lesion and 5 patients $(12.5 \%)$ presented as non mass like enhancement. MRI description of non mass like enhancement showing non mass like enhancement, 1 patient presented with ductal enhancement, 1 with segmental, 1 with regional and 2 with multiregional enhancement.

\begin{tabular}{ll}
\hline \multicolumn{2}{l}{ Table 1: BIRADS lexicon and enhancement } \\
\hline Type of lesion by BIRADS lexicon & No. of lesions \\
\hline Focus / Foci & 0 \\
Mass & $35(87.5 \%)$ \\
Non mass like enhancement & $5(12.5 \%)$ \\
\hline Non mass like enhancement & \\
Focal & 0 \\
Linear/Ductal & 1 \\
Segmental & 1 \\
Regional & 1 \\
Multiregional & 2 \\
Diffuse & 0 \\
\hline
\end{tabular}

Out of 19 benign lesions $8(42 \%)$ had round shape and $8(42 \%)$ had oval shape. Out of 16 malignant lesions 12 (75\%) had irregular shape. Out of 19 benign lesions, $16(82 \%)$ had well circumscribed margins.

Out of 16 malignant lesions 10 (62.5\%) had spiculated margins [Table 2].

Out of 33, 14 lesions (42.5\%) had homogenous enhancement which are mostly benign. Five lesions showed dark internal septations which were all benign. 11 lesions (33.5\%) showed heterogenous enhancement which are mostly malignant. 
Table 2: Mass morphologic descriptors and Kinetic Curve Assessment.

\begin{tabular}{lll}
\hline Descriptor & $\begin{array}{l}\text { Benign } \\
\text { lesions }\end{array}$ & $\begin{array}{l}\text { Malignant } \\
\text { lesions }\end{array}$ \\
\hline $\begin{array}{l}\text { Descriptor (shape) } \\
\text { Round }\end{array}$ & 8 & 1 \\
Oval & 8 & 3 \\
Irregular & 3 & 12 \\
Total & 19 & 16 \\
Descriptor (margin) & & \\
Circumscribed Irreg- & 16 & 2 \\
ular Spiculated & 1 & 4 \\
& 2 & 10 \\
Total & 19 & 16 \\
\hline
\end{tabular}

Early rim enhancement is seen in 3 lesions (9\%). Out of which two of them were proven to be malignant and one lesion was an infective abscess.

Out of 40 lesions, 22 were benign, when analysed by Kinetic Curve Assessment 17 lesions (77.3\%) showed Type I curve i.e., progressive pattern.5 lesions (22.7\%) showed Type II curve i.e., plateau pattern. None of them showed Type III curve i.e., washout pattern [Table 3].

In 18 malignant lesions 10 lesions (55.6\%) showed Type III curve, 7 lesions (38.9\%) showed Type II curve, 1 lesion $(5.6 \%)$ showed Type I curve.

Table 3: Assessment of Kinetic Curve in benign and malignant lesions

\begin{tabular}{|lll|}
\hline Type of curve & Benign lesions & $\begin{array}{l}\text { Malignant } \\
\text { lesions }\end{array}$ \\
\hline Type I & 17 & 1 \\
\hline Type II & 5 & 7 \\
Type III & 0 & 10 \\
\hline Total & 22 & 18 \\
\hline
\end{tabular}

Table 4: BI-RADS assessment Category

\begin{tabular}{ll}
\hline BI-RADS Category & No. of lesions \\
\hline Category 0 & 0 \\
\hline Category 1 & 0 \\
\hline Category 2 & 7 \\
Category 3 & 12 \\
Category 4 & 10 \\
Category 5 & 11 \\
\hline Category 6 & 0 \\
\hline
\end{tabular}

\section{Histopathology of lesions:}

Table 5: Histopathology of lesions

\begin{tabular}{ll}
\hline Histology / Cytology & No. of lesions \\
\hline 1) Malignant & \\
\hline i) Invasive Breast Cancer & 13 \\
Ductal & 2 \\
\hline Lobular & 0 \\
Medullary & 0 \\
Mucinous & \\
\hline ii) Non-Invasive Breast Cancer & 2 \\
\hline Ductal Carcinoma In Situ & 1 \\
\hline Intracystic Papillary carcinoma & \\
\hline 2) Benign & 8 \\
\hline Fibroadenoma & 1 \\
\hline Intracystic Papilloma & 5 \\
\hline Fibrocystic disease & 1 \\
\hline Pagets disease & 1 \\
\hline Phyllodes tumor & 1 \\
\hline Abscess & 2 \\
\hline Infective Etiology & \\
\hline
\end{tabular}

Invasive Duct Cell Carcinoma was the most common histology in the malignant breast lesions in our study, seen in 13 out of $18(72.2 \%)$.

Fibroadenoma was the most common benign breast lesion, observed in 8 out of $19(42.1 \%)$.

\section{Post operative Histopathalogical Lesions :}

In 4 post operative patients ,2 (50\%) were post operative fibrosis, 1 (25\%) was recurrance and 1 (25\%) was post operative collection.

\begin{tabular}{lll}
\hline Table 6: Associated findings & & \\
\hline Associated findings & Benign & Malignant \\
\hline Nill & 14 & 6 \\
Nipple Retraction & 0 & 2 \\
Nipple Invasion & 1 & 0 \\
Skin Retraction & 1 & 11 \\
Skin Thickening & 3 & 10 \\
Axillary Lymphhadenopa- & 4 & 9 \\
thy & & \\
\hline Pectoralis Involvement & 0 & 1 \\
Architectural Distortion & 0 & 1 \\
\hline Cysts & 5 & 0 \\
\hline
\end{tabular}

In 18 malignant lesions, 11 patients (61.1\%) had skin retraction, 10 patients $(55.5 \%)$ had skin thickening, 9 patients 
(50\%) had axillary lymphadenopathy, 2 patients $(11.1 \%)$ had nipple retraction, one patient $(5.6 \%)$ had pectoralis involvement.

In 22 benign lesions, 5 patients $(22.7 \%)$ had cysts, 4 patients $(18.2 \%)$ had axillary lymphadenopathy.

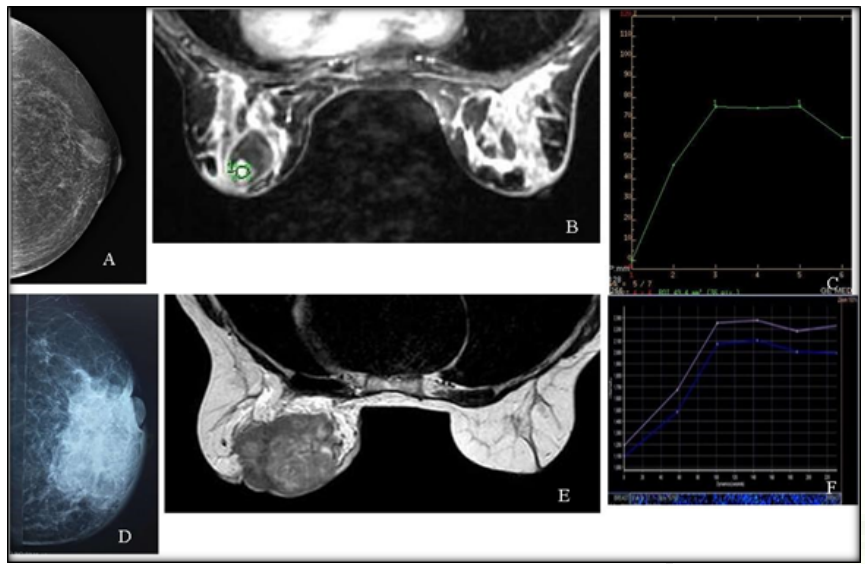

Figure 1: Patient-2: Intracystic Papillary Carcinoma. A. Left breast mammogram. B. DCE MRI-Post contrast axial T1W image C. kinetic curve. (patient 2) D.Patient 4. Inflammatory Ductal Cell Carinoma . Left breast mammogram. E. Pre contrast axial T2W image. F. kinetic curve.

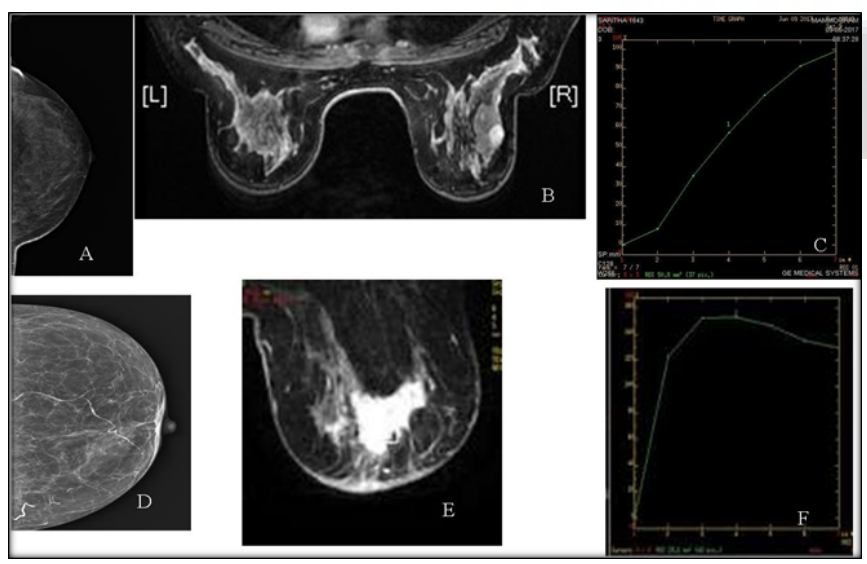

Figure 2: Patient 6: Fibroadenoma A. mammogram of right breast. B. Post contrast axial T1W image. C. kinetic curve. D. Patient 7-Invasive Lobular Carcinoma. Mammogram of right breast. E. Post contrast axial T1W image. F. dynamic curve.

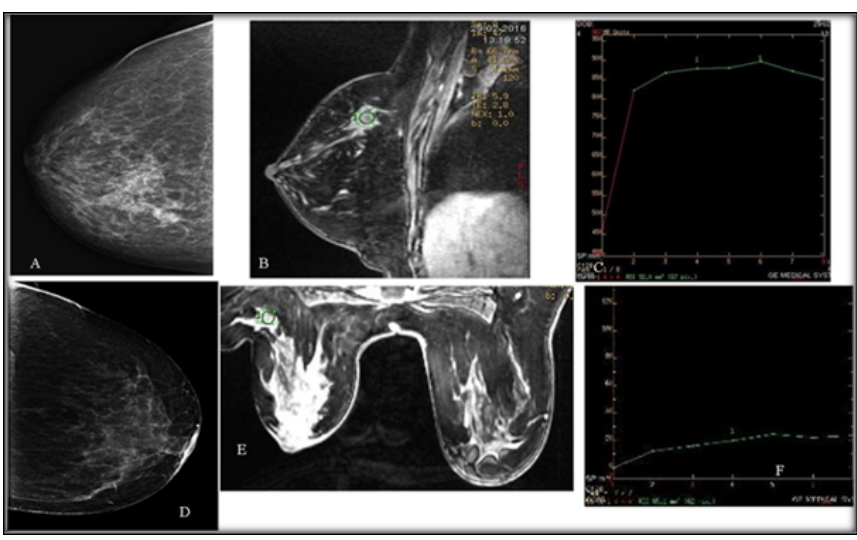

Figure 3: A. Patient 8- Invasive Ductal Cell Carcinoma. Mammogram right breast. B. Post contrast sagittal T1W image C. Dynamic curve. D. Patient 9 . Infective etiology (koch's) . Mammogram of left breast. E. Post contrast axial T1W image. F. kinetic curve

\section{Discussion}

The sensitivity of MR imaging for detection of breast cancer is very high, and approaches $100 \%$ for invasive carcinoma.

In our study we found that morphological appearance of lesion on post contrast study and qualitative assessment of time signal intensity curves are most useful imaging parameters for breast MRI and our results are comparable to previous tudiest. ${ }^{[6,7]}$

\section{Morphological Analysis:}

Out of 40 patients, 35 patients $(87.5 \%)$ presented as mass lesions and 5 patients $(12.5 \%)$ presented as non masslike enhancement. Out of 5 patients showing non mass-like enhancement, 1 patient presented with ductal enhancement, 1 with segmental enhancement, 1 with regional enhancement and 2 with multi regional enhancement.

In 35 patients presented as mass lesions, 19 patients has histological diagnosis of benign disease. Out of 19 patients, 5 patients had fibrocystic change. All these patients showed multiple well defined rounded cysts with no or minimal enhancement of cyst wall on contrast administration. Morphologic features in MRI nearly had $100 \%$ NPV for diagnosis of malignancy in fibrocystic change.

Eight patients had histopathological diagnosis of Fibroadenoma, in these 5 lesions (62.5\%) showed dark non enhancing internal septations. So, dark non enhancing internal septations had 100\% PPV for Fibroadenoma).

Kuhl et al., ${ }^{[8]}$ reported that dark septation if present within a lobular oroval mass are typical of fibroadenomas. 
In 35 patients presented as mass lesions, 16 patients had malignant histology. In these 12 lesions (75\%) had irregular shape and 10 patients $(62.5 \%)$ had spiculated margins.

Hence the sensitivity of irregular shape and spiculated margins for malignant disease are $75 \%$ and $62.5 \%$ respectively.

Out of 12 patients with spiculated margins, 10 patients has malignant histology and out of 15 lesions with irregular shapes, 12 had malignant histology. So PPV of spiculated margins and irregular margins for malignant disease are 83.3\% and $80 \%$ respectively.

Turnbull et al. ${ }^{[9]}$ reported that the most frequent morphological finding among the malignant lesions was heterogeneous internal enhancement.

In our study 11 lesions showed heterogenous enhancement which are mostly malignant.

In our study 14 lesions had homogenous enhancement which are mostly benign. 5 lesions showed dark internal enhancement which were all benign. 3 lesions showed rim enhancement which were all malignant.

Lesions showed heterogenous enhancement which are mostly malignant

Data from American College of Radiology BI-RADS and Numes et al, update of breast MR imaging architectural interpretation model, 219:484-94 shown following PPV for different morphologic features. ${ }^{[10]}$

Table 7: Positive predictive value of morphologic features for malignancy

\begin{tabular}{|lll|}
\hline Feature & \%PPV & $\begin{array}{l}\text { PPV of present } \\
\text { study }\end{array}$ \\
\hline $\begin{array}{l}\text { Round mass with non- } \\
\begin{array}{l}\text { Mass } \\
\text { enhancing internal } \\
\text { septations }\end{array}\end{array}$ & 6.25 \\
\hline $\begin{array}{l}\text { Ductal enhancement } \\
\text { Rim enhancement }\end{array}$ & $24-85$ & - \\
\hline Irregular margin & $40-86$ & 66.6 \\
\hline Spiculated margin & $32-84$ & 25 \\
\hline
\end{tabular}

Present study had low \%PPV for irregular margins $(25 \%)$ and spiculated margins $(62.5 \%)$. This could be due small sample size, it is not the representative sample of general population. This study correlated for $\% \mathrm{PPV}$ for rim enhancement (66.6\%) which is between $40 \%-86 \%$.

\section{Kinetic Curve Assessment:}

Our study revealed that the enhancement kinetics are shown by the time signal intensity curves differ significantly for benign and malignant enhancing lesions, so can be used as an aid in differential diagnosis. In malignancies washout and plateau curves prevail and benign lesions show progressive enhancement.

In our study group of 40 patients, 22 patients had histopathalogical diagnosis of benign disease, when analysed by Kinetic curve assessment, 17 lesions (77.2\%) showed Type 1 curve i.e., progressive pattern, 5 lesions (22.7\%) showed Type 2 curve i.e., plateau pattern and no lesions showed Type 3 curve. In 18 malignant lesions, 10 lesions (55.5\%) showed Type 3 curve i.e., washout pattern, 7 lesions (38.9\%) showed Type 2 curve i.e., plateau pattern and 1 lesion (5.5\%) showed Type 1 curve i.e., progressive pattern.

In Susan et al. ${ }^{[11]}$ study group, $83 \%$ of the benign lesions exhibited a steady or curved time-signal intensity curve. In contrast $57 \%$ of malignant lesions exhibited a washout timesignal intensity curve. Using the shape of time signal intensity curve alone, the authors reported a sensitivity of 91\%(92 of 101 ), a specificity of $83 \%(137$ of 165$)$, a positive predictive value of 77\%(92 of 120), a negative predictive value of $94 \%$ (137 of 146) and a diagnostic accuracy of 86\%(229 of 266). The likelihood of breast cancer associated with Type 1,2 or 3 time curves was $6 \%$ ( 9 of 146), 64\% (34 of 53) and 87\% (58 of 67) respectively.

\section{Involvement of skin, pectoralis muscle, or chest wall:}

In our study out of 18 malignant lesions, 11 patients (61.1\%) had skin retraction, 10 patients $(55.5 \%)$ had skin thickening, 9 patients $(50 \%)$ had axillary lymphadenopathy, 2 patients (11.1\%) had nipple retraction and 1 patient $(5.6 \%)$ had pectoralis involvement. Out of 22 benign lesions, 5 patients (22.7\%) had cysts and 4 patients (18.2\%) had axillary lymphadenopathy.

Similar results were shown by Morris and colleagues reported on 19 women who had posterior enhancing breast masses at preoperative breast MR imaging. ${ }^{[12]}$ Hence it's concluded that violation of the fat plane without other findings did not indicate muscle or chest wall involvement; extension of tumor into underlying chest wall or muscle was indicated by abnormal enhancement within these deep structures .

\section{Post Operative Scar Vs Recurrence}

In our study out of 4 post operative lesions, $1(25 \%)$ was recurrent lesion, $2(50 \%)$ were scar tissue and $1(25 \%)$ was post operative seroma. With DCE-MRI we can diagnose with $100 \%$ sensitivity for scar tissue and recurrent lesions.

Giles R, showed nodular enhancement in all cases of invasive carcinoma and linear enhancement was observed in cases of DCIS recurrence. ${ }^{[13]}$ The majority of scars showed no 
enhancement after major breast surgery, fresh scars may exhibit contrast enhancement. Scar enhancement is relatively subtle and occurs more or less evenly in the entire scar. This is in contrast to recurrent cancer, which exhibits a mass effect within the scar and causes focal enhancement that is confined to a part of the scar.

Both malignant and benign lesions are identified with high resolution MR imaging. Malignant lesions tend to have an irregular and spiculated margins and demonstrated heterogenous and peripheral rim enhancement. Fibroadenomas cocnsistently have lobulated and well defined rounded or ovoid lesions with smooth margins and non enhancing internal septations as specific morphologic feature. MRI offers superb visualisation of the posterior breast tissue can assess contiguous involvement better than conventional imaging, and also detects multifocal or multicentric lesions. Routinely we do bilateral imaging for breast MRI, by this we can detect synchronous lesions also. So, helps in pre operative staging of malignant lesions and provides better planning and management of the breast lesions. Surgical outcomes proved to be best with the knowledge of the tumor extent, multi centricity, multi focality and synchronous breast lesions.

\section{Conclusion}

Dynamic contrast enhanced MRI is a valuable tool in evaluation of postoperative breast as it has high specificity in differentiating the postoperative changes. Breast MRI minimizes unnecessary intervention and optimizes diagnosis of recurrence in its early stages.

In our study, MR imaging alone has Sensitivity of $88.8 \%$, Specificity of $86.3 \%$, Positive Predictive Value of $84.2 \%$, and Negative Predictive Value of $90.4 \%$ and Accuracy of $87.5 \%$. By combining kinetic analysis with morphological analysis we had additional benefit to correctly categorise the breast lesions into benign and malignant and that increased the confidence of diagnosis. CE-MRI with both morphology and kinetic curve assessment has a Sensitivity of $94.5 \%$, Specificity of $100 \%$ and Accuracy of $97.5 \%$.

\section{References}

1. Schnall MD. Breast MR imaging. Radiol Clin North Am. 2003;41(1):43-50. Available from: https://dx.doi.org/10.1016/ s0033-8389(03)00068-4.

2. Sylvia H. Heywang - kobrunner and petranehweg et al; Text book of. Magnetic Resonance Imaging;1(2):307-320.
3. Stavros AT, Thickman D, Rapp CL, Dennis MA, Parker SH, Sisney GA. Solid breast nodules: use of sonography to distinguish between benign and malignant lesions. Radiology. 1995;196(1):123-134. Available from: https://dx.doi.org/10. 1148/radiology.196.1.7784555.

4. Agoston AT, Daniel BL, Herfkens RJ. Intensity modulated parametric mapping for simultaneous display of rapid dynamic and high spatial resolution breast MR Imaging data. Radiographics. 2001;21(1):217-226. Available from: https://doi.org/ 10.1148/radiographics.21.1.g01ja22217.

5. Rahbar H, Partridge SC. Multiparametric Breast MRI of Breast Cancer. Magn Reson Imaging Clin N Am. 2016;24(1):223238. Available from: https://dx.doi.org/10.1016/j.mric.2015. 08.012 .

6. Khatri VP, Stuppino JJ, Espinosa MH, Pollack MS. Improved accuracy in differentiating malignant from benign mammographic abnormalities. Cancer. 2001;92(3):471-478. Available from: https://dx.doi.org/10.1002/1097-0142(20010801) 92:3<471::aid-cncr1345>3.0.co;2-6.

7. Siegman KC, Schimfle MM, Shick F, Remy TC. MR imaging detected breast lesions: histopathological correlation of lesion characteristic and signal intensity data. Am J Roentgenol. 2002; 178:1403-1412.

8. Kuhl CK. Concepts for differential diagnosis in breast MR imaging. Magn Reson Imaging Clin N Am. 2006;14(3):305328. Available from: https://doi.org/10.1016/j.mric.2006.07. 002.

9. Turnbull L, Brown S, Harvey I, Olivier C, Drew P, Napp $\mathrm{V}$, et al. Comparative effectiveness of MRI in breast cancer (COMICE) trial: a randomised controlled trial. Lancet. 2010;375(9714):563-571. Available from: https://dx.doi.org/ 10.1016/s0140-6736(09)62070-5.

10. Nunes LW, Schnall MD, Orel SG, Hochman MG, Langlotz $\mathrm{CP}$, Reynolds CA, et al. Breast MR imaging: interpretation model. Radiology. 1997;202(3):833-841. Available from: https://dx.doi.org/10.1148/radiology.202.3.9051042.

11. Orel SG, Weinstein SP, Schnall MD, Reynolds CA, Schuchter LM, Fraker DL, et al. Breast MR imaging in patients with axillary node metastases and unknown primary malignancy. Radiology. 1992;212(2):543-549. Available from: https://doi. org/10.1148/radiology.212.2.r99au40543.

12. Morris EA, Schwartz LH, Drotman MB, Kim SJ, Tan LK, Liberman L, et al. Evaluation of Pectoralis Major Muscle in Patients with Posterior Breast Tumors on Breast MR Images: Early Experience. Radiology. 2000;214(1):67-72. Available from: https://dx.doi.org/10.1148/radiology.214.1.r00ja1667.

13. Gilles R, Guinebretière JM, Toussaint $\mathrm{C}$, Spielman M, Rietjens M, Petit JY, et al. Locally advanced breast cancer: contrastenhanced subtraction MR imaging of response to preoperative chemotherapy. Radiology. 1994;191(3):633-638. Available from: https://dx.doi.org/10.1148/radiology.191.3.8184039. 
Copyright: (C) the author(s), 2020. It is an open-access article distributed under the terms of the Creative Commons Attribution License (CC BY 4.0), which permits authors to retain ownership of the copyright for their content, and allow anyone to download, reuse, reprint, modify, distribute and/or copy the content as long as the original authors and source are cited.

How to cite this article: Veena $\mathrm{KH}$, Baru RR. Role of Dynamic Contrast Enhanced Magnetic Resonance Imaging and Mammogram in Evaluation of Breast Lesions with Pathological Correlation. Asian J. Med. Radiol. Res. 2020;8(2):112-118.

DOI: dx.doi.org/10.47009/ajmrr.2020.8.2.18

Source of Support: Nil, Conflict of Interest: None declared. 